\title{
Utility of Interferon Gamma Assays and Comparison with Tuberculin Skin Test in Rheumatoid Arthritis Patients
}

\author{
Shradha $B^{1}$, Arun $C^{2}$, Jyostna $J^{3}$ and Sudha $S^{1^{*}}$ \\ ${ }^{1}$ Sir H. N Medical Research Society. \\ ${ }^{2}$ Sir H. N. Reliance Foundation Hospital and Research Centre, Raja Ram Mohan Roy Road, \\ Prarthana Samaj, Girgaum, Mumbai 400004, Maharashtra, India. \\ ${ }^{3}$ Deptment of Chest Medicine, Topiwala National Medical College and B Y L Nair Hospital, Mumbai.
}

Received: March 15, 2018; Accepted: April 13, 2018; Published: April 20, 2018

*Corresponding author: Sudha S Deo, Principal Scientist, Sir H. N Medical Research Society, Sir H. N. Reliance Foundation Hospital and Research Centre, Raja Ram Mohan Roy Road, Prarthana Samaj, Girgaum, Mumbai 400004, Maharashtra, India. Tel: +91-022-35553886; E-mail:sudha.deo@ rfhospital.org

\begin{abstract}
Patients with Rheumatoid Arthritis (RA) are at higher risk of developing tuberculosis due to treatment with immunosuppressive drugs.
\end{abstract}

Objective: The objective of this study was to investigate the agreement between the interferon gamma release assays (IGRAs) and Tuberculin Skin Test (TST) in the Rheumatoid Arthritis (RA) patients compared with the Controls.

Methods: 42 consecutive RA patients diagnosed according to the 1987 ACR criteria were recruited for our study. 50 culture-positive Tuberculosis (TB) patients were enrolled and 50 Healthy Controls were taken for comparison. QFT-GIT assay, Elispot Plus assay and TST were performed on all the study participants. BCG vaccination status and current medication was noted.

Result: The QFT-GIT and TST demonstrated $60.71 \%$ agreement; $\mathrm{k}=0.313$ in patients with RA and $70.83 \%$ agreement; $\mathrm{k}=0.408$ in healthy controls. The Elispot assay and TST demonstrated $53.57 \%$ agreement; $\mathrm{k}=0.308$ in patients with RA and $79.17 \%$ agreement; $\mathrm{k}=$ 0.565 in healthy controls. There is a moderate agreement in between the two IGRAs (78.5\%; $\mathrm{k}=0.593$ ). The sensitivity of TST and IGRA was equally low, but the specificity of IGRA was higher than TST.

Conclusion: Our study showed low moderate agreement between the results of the interferon gamma release assays (IGRAs) and the TST in RA patients, but in controls, moderate agreement was observed between the tests.

Keywords: QFT-GIT, Elispot, TST, LTBI, RA.

\section{Introduction}

Rheumatoid arthritis (RA) is a systemic autoimmune disease which leads to swelling (inflammation) of various joints. The swelling destroys joints from within and causes permanent deformities. It affects about $0.92 \%$ of adult population in India. The prevalence of rheumatoid arthritis in Indian population have been estimated to vary between $0.3 \%$ to $0.75 \%$ and based upon 2011 census, more than 36 million patients are expected to be suffering from rheumatoid arthritis in India. [1]A study in Mumbai published in 2008 by Tembe et al has reported a high F: $\mathrm{M}$ ratio in their study population, wherein $88.5 \%$ were females. [2] The risk of latent tuberculosis reactivation in RA patients is a perpetual concern among rheumatologists. Patients with RA have a 4-fold increased risk of tuberculosis infection compared with the general population. [3] The accurate diagnosis of latent tuberculosis infection (LTBI) has become an important issue in the field of rheumatology. [4] Active TB is believed to develop due to reactivation of latent tuberculosis infection. LTBI is defined in the World Health Organisation (WHO) guidelines as a state of persistent immune response to stimulation by Mycobacterium tuberculosis antigens without evidence of clinically manifested active TB.[5]

According to the World Health Organisation (WHO) Global TB report, 2015 approximately one-third of the world population is infected with Mycobacterium tuberculosis (MTb).[6] India is the highest TB-burden country accounting for one-fifth (21\%) of the global incidence.[7] Being TB endemic country, Bacillus Calmette-Guerin (BCG) is mandatory at birth in India. Infections like tuberculosis are a leading cause of morbidity and mortality in rheumatoid arthritis patients. Since many years, TST was the only available tool for the detection of LTBI despite its lack of specificity due to cross-reactivity with the BCG vaccine and other non-tuberculosis mycobacterium and its low sensitivity in immuno compromised patients.[8] This poor sensitivity can lead to false-negative results, with a subsequent risk of TB reactivation following anti-TNF $\alpha$ therapy. There is a need for a more accurate test for MTb infection in patients with RA. [9] TST requires a return visit to read the test and it may produce false-positive results owing to prior BCG vaccination or non tuberculosis mycobacterial infection, this poor specificity can lead to unnecessary LTBI treatment, with the risk of drug toxicity. [10] 
There is no gold standard for LTBI. Currently, two vastly different methods of testing are available: the traditional Tuberculin Skin Test (TST) and the newly developed Interferon Gamma Release Assays (IGRAs). The TST measures type IV hypersensitivity in response to purified protein derivative (PPD), which contains a mixture of antigens of Mycobacterium tuberculosis that are also present in Mycobacterium bovis BCG, and other non tuberculous mycobacteria. [3] The development of interferon-gamma release assays (IGRAs) is an important advance in the diagnosis of latent tuberculosis infection (LTBI). IGRAs are in vitro blood tests of cell-mediated immune response; they measure $\mathrm{T}$ cell release of interferon (IFN)-gamma following stimulation by antigens unique to Mycobacterium tuberculosis. [11] IGRAs cannot distinguish between latent infection and active tuberculosis (TB) disease and should not be used for diagnosis of active TB, which is a microbiological diagnosis. IGRAs have been approved by the CDC as an alternative screening strategy to TST for the diagnosis of LTBI. Some current national guidelines for screening prior to anti-TNF treatment recommend the use of the IGRA instead of the TST. [10]

A particular concern in immune mediated inflammatory diseases is that the TST may also be modified by concomitant immunosuppressive drugs and produce false-negative results, exposing patients to the risk of TB reactivation when treated with anti-TNF therapy. [12] Because IGRAs are not affected by Bacille Calmette-Guérin (BCG) vaccination status, IGRAs are useful for evaluation of LTBI in BCG-vaccinated individuals, particularly in settings where BCG vaccination is administered after infancy or multiple (booster) BCG vaccinations are given.[12] Neither the TST nor IGRAs have been proven to be $100 \%$ accurate. TST's main weakness appears to be its low sensitivity in immuno suppressed patients because of their deficient cell-mediated immunity and \or chronic use of immuno suppressents. [3] An important concern for patients with rheumatic disease is that the immuno suppression due to disease itself and TST will impact on the performance of both tests. It has been shown that TST is more likely to produce false negative result in rheumatic patients than the general population due to the weakened cellular immune response. [13]

Anti-tumor necrosis factor-alpha (TNF- $\alpha$ ) agents are increasingly being used to treat RA. However, they have been reported to increase the risk of infection, and in particular, to reactivate latent infections. A temporal association between anti-TNF- $\alpha$ antibodies and reactivation of latent tuberculosis infection (LTBI) has been established. TNF- $\alpha$ is one of the key molecules involved in granuloma formation and maintenance of TB infection. [14] Guidelines have been developed in many countries for screening for latent tuberculosis before starting TNF- $\alpha$ antagonists. [15] Thus, the detection and treatment of LTBI by prophylactic therapy are essential before prescribing antiTNF agents, because this screening has reduced the incidence of TB.[16] Identification of genes in the TB genome that are absent in BCG and most environmental mycobacterium offers an opportunity to develop more specific tests to investigate TB infection, particularly LTBI.[17] Advances in mycobacterial genomics have led to the development of 2 new blood interferon- $\gamma$ release assays (IGRA) in response to 3 unique antigens, ESAT-6, CFP- 10 and TB 7.7 that are highly specific for M. tuberculosis, and which are absent from M. bovis, M. avium, and most other non tuberculosis mycobacteria. One assay, the enzyme-linked immunospot (Elispot Plus, Mabtech) enumerates IFN- $\gamma$-secreting $\mathrm{T}$ cells; the other measures IFN- $\gamma$ concentration in supernatant by Enzyme Linked Immunosorbent Assay (ELISA) (QuantiFeronTB Gold In-Tube; Cellestis, Carnegie, Australia); the QuantiFeronTB Gold In-Tube (QFT-GIT) assay (Cellestis Limited) has replaced the second-generation QuantiFeron-TB Gold (QFT-G) assay. The usefulness of interferon $r$ release assays (IGRAs) has been evaluated in populations with a moderate or high prevalence of TB disease, in contact tracing after fresh TB outbreaks and in patients receiving immunosuppressive therapy for systemic autoimmune disorders. These studies have consistently demonstrated that IGRAs are sensitive and have a higher specificity for TB infection than TSTs, irrespective of BCG vaccination status. A high level of concordance between different IGRAs was generally seen.[12] The aim of our study were to compare the prevalence of LTBI in RA patients and age matched controls as estimated by TST and positivity by two interferon gamma release assays (IGRAs), and to determine the level of agreement between these tests.

\section{Materials and Methods}

In this study, 42 consecutive patients with RA, diagnosed by American College of Rheumatology (ACR) criteria and after radiological analysis, were included in the study. In the event of abnormal X-ray of Chest, HRCT of chest was done for identification of infections. Recruitment of the patients was from OPD of Sir H. N. Reliance Foundation Hospital and Research Centre, Mumbai. 50 patients with Tuberculosis were recruited from Department of Chest Medicine, TN Medical College and B Y L Nair Hospital, Mumbai. The Control group of 50 Healthy participants of similar age were also enrolled. BCG status was determined for all the study participants. HIV testing was done in a diagnostic laboratory by rapid immunoassay method.

\section{RA patients}

All RA patients were evaluated for their systematic involvement. Besides this, at the time of recruitment, the physical findings such as height, weight, blood pressure, duration of the disease was done. DAS28-CRP score was calculated; RF test and CRP analysis was done. The treatment for RA patients was recorded. All patients also underwent a radiological examination of the Chest. The patients below 18 years of age, pregnant patients, HIV positive patients, hospitalized patients and patients with past history of infection in last one year were excluded from the study.

\section{Healthy Controls}

This group consisted of healthy adult subjects age $\geq 18$ years who did not show signs of auto-immune disease, apparently free of TB symptoms. All the subjects were recruited from offices, colleges, Security staff and relatives $\backslash$ friends of RA patients. Since our setting is endemic to TB, to rule out the suspicion of 
active TB disease, all the subjects were subjected to radiological examination.

\section{TB patients}

This group consisted of pulmonary TB patients who were recruited from TN Medical College and B Y L Nair Hospital, Mumbai. All the TB patients were negative for HIV testing. These patients attended the OPD of Chest Medicine, Nair Hospital with abnormalities, suggestive of pulmonary tuberculosis (PTB) in the Chest X-ray and positive results for sputum microscopy. Three sputum samples were collected from these patients and all were subjected to radiological examination. The collected sputum samples were processed, stained for acid fast bacilli (AFB) microscopy by Zeil-Neelsen method and cultured in Bactec MGIT 960 TB system at the Microbiology Department, Hinduja Hospital \& Research Centre, Mumbai. The presence of active TB was defined as positive for sputum smear microscopy, positive for M. tuberculosis in sputum culture and abnormality suggestive of TB in chest X-ray.

\section{LTBI screening}

All the patients underwent LTBI screening which included a detailed history of TB exposure and a Chest radiograph. On radiographic examination, features considered as suggestive of previous infection were pulmonary nodules, upper lobe bronchiectasis, apical pleural thickening, interstitial granulomatous calcifications, cavitations, and lymph node or pericardial calcifications. [18]

\section{Methods}

$10 \mathrm{ml}$ of blood was collected through peripheral venipuncture from all the study participants. The serum was separated and stored at $-80^{\circ} \mathrm{C}$ for determination of biochemical parameters. QFT-Gold in Tube (QFT-GIT) Elisa kit: Cellestis, Carnegie, Australia; and enzyme-linked immunospot (ELISpot Plus for Human INF-gamma, from Mabtech) were used for determination of the interferon gamma levels in all the study participants. TST was also performed on all the study participants.

\section{Interferon Gamma Release Assays (IGRAs)}

Prior to TST screening, peripheral venous blood samples for the two IGRAs (Elispot and QFT-GIT) were collected simultaneously and processed according to the manufacturer's recommendations within $2 \mathrm{hr}$. Each test included a negative control (Nil), a positive control (Mitogen) and MTb specific antigens (ESAT-6, CFP-10 and TB 7.7).

\section{Elispot Plus Assay}

The supernatant plasma was separated after overnight incubation at $37^{\circ} \mathrm{C}$, by centrifugation from the 3 tubes- Antigen, Mitogen and Nil. The supernatant plasma will be processed by QFT-GIT.

ELISPOT plates precoated with monoclonal antibody to interferon-gamma (Mabtech $\mathrm{AB}$, Stockholm, Sweden) were used. Briefly, the whitish layer of cells obtained after overnight incubation from the tubes, were layered over a Ficoll gradient and then centrifuged for 12 mins at $1500 \mathrm{rpm}$ to get the peripheral blood mononuclear cells (PBMC). The cells were then suspended in complete RPMI media which consists of RPMI 1640 medium (Sigma Aldrich) plus 10\% fetal bovine serum (FBS, Himedia). Plates were seeded with $2.5 \times 10^{5}$ peripheral blood mononuclear cells per well. After overnight incubation at $37^{\circ} \mathrm{C}, 5 \%$ carbon dioxide, the wells were washed and developed with a conjugate against the antibody used and an enzyme substrate. Assays were scored by automated ELISPOT reader (Immunospot, Cellular Technology Limited, USA). The responses were considered positive if the test wells contained a higher mean number of spotforming cells (SFC) than the mean number plus two standard deviations (SD) in the negative control wells and when the number of SFC per million PBMC in the stimulated wells (minus the values of the negative control wells) was $\geq 20$. (Figure 1) When the mean number of SFC per million PBMC in the positive control wells was $<100$, the responses in the test wells were considered intermediate.

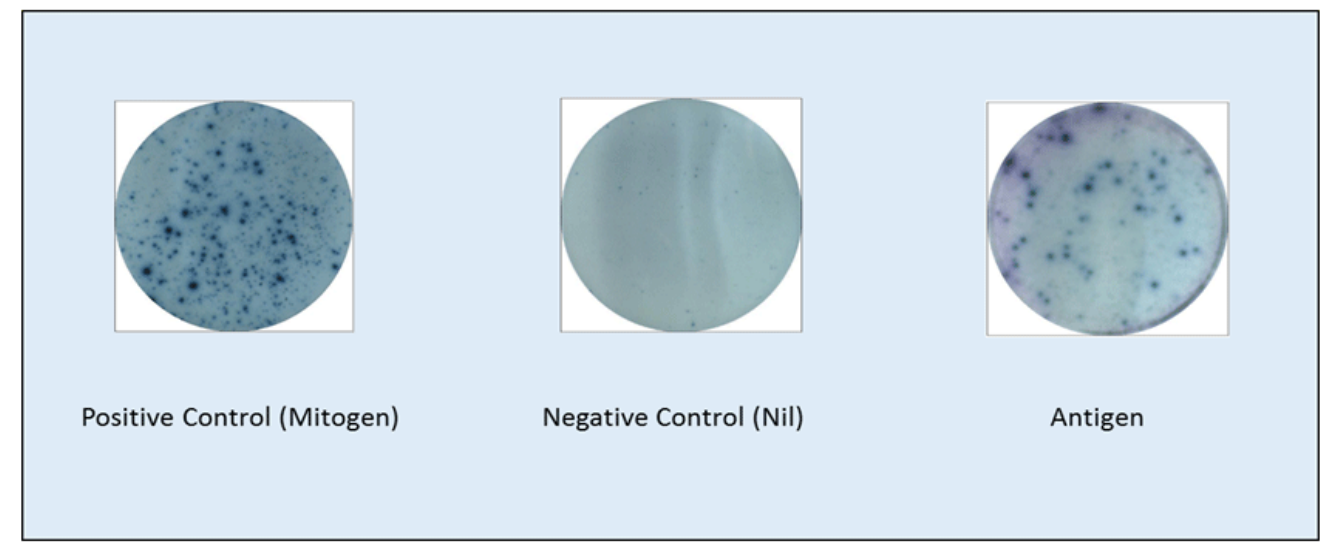

Figure 1: Image of the well as seen in an Elispot Reader: Example of a positive result of an Elispot assay for a patient. 


\section{QFT-Gold in Tube Test}

The QFT-Gold in Tube (QFT-GIT) test was performed at the laboratory according to the manufacturer's recommendations. Briefly, whole blood specimens were incubated for 20 hours (overnight) at $37^{\circ} \mathrm{C}$ in a humidified atmosphere. The plasma was harvested and stored frozen at $-20^{\circ} \mathrm{C}$ until further analysis. The concentration of IFN- $\gamma$ present in each plasma was determined using the QFT ELISA. The IFN $\gamma$ level of a nil well was considered background and was subtracted from the results of the mitogen well and the antigen-stimulated wells. The test result was considered positive if the concentration of IFN- $\gamma$ in the sample well after stimulation with early secretory antigen target 6 , culture filtrate protein 10 and TB 7.7 was $\geq 0.35 \mathrm{IU} / \mathrm{mL}$ (after subtracting the nil well value), regardless of the result of the positive control (mitogen well). The test result was considered negative if the response to the specific antigens (after subtraction of the nil well value) was $<0.35 \mathrm{IU} / \mathrm{mL}$ and if the IFN- $\gamma$ level of the positive control (after subtraction of the nil well value) was $\geq 0.5 \mathrm{IU} / \mathrm{mL}$. The test result was considered indeterminate if both antigen-stimulated sample wells were negative $(<0.35 \mathrm{IU} /$ $\mathrm{mL}$ after subtraction of the nil well value) and if the value of the positive control well was $<0.5 \mathrm{IU} / \mathrm{mL}$ (after subtraction of the nil well value). Repeat testing of the indeterminate results were done.

\section{Tuberculin Skin Test (TST)}

A 5-TU dose of PPD RT 23 (Statens Serum Institute, Copenhagen, Denmark) was injected intradermally by Mantoux method and the induration was measured between 48-72 hrs after injection by trained professionals. The cut-off point for TST positivity was considered as $10 \mathrm{~mm}$ for this study.

\section{Ethics Consideration}

This study was approved by the Scientific Advisory Committee and the Institutional Ethics Committee of Sir H. N. Reliance Foundation Hospital and Research Centre and informed consent was taken from the patient before the collection of their samples. The study was carried out in accordance with the "Ethical Guidelines for Biomedical Research on Human Participants, 2006" by the Indian Council of Medical Research and the Declaration of Helsinki, 2008.

\section{Statistical Analyses}

The analyses was performed using the Statistical Package for Social Sciences SPSS) software, version 21.0 (SPSS, Chicago, IL, USA). The numerical data confirmed to a normal distribution was assessed by Kolmogorov-Smirnov test. Independent t-test was used to evaluate the differences between patients and Controls. The significance cut-off value (P) was fixed to 0.05 . Percentage agreement between IGRAs and TST was calculated and the $\mathrm{k}$ statistic was generated. The kappa coefficient $(\mathrm{k})$ was calculated to determine the concordance between the tests. The strength of the agreement was considered "poor" for $\mathrm{k} \leq 0.20$, "low-moderate" for $0.20 \leq \mathrm{k} \leq 0.40$, moderate for $0.40 \leq \mathrm{k} \leq 0.6$, "substantial" for $0.6 \leq \mathrm{k} \leq 0.80$, and "optimal" for $0.8 \leq \mathrm{k} \leq 1$. Chi-square test was used to calculate the sensitivity and the specificity for the tests.

\section{Results}

\section{Study Population}

As shown in Table 1, 42 RA patients were recruited, of which 4 patients showed anergy reactions. So 38 patients were included in the study. Table 1 shows the data of 38 RA patients with mean age of $45 \pm 12.12$ yrs; $39.4 \%$ showed having history of Tuberculosis; BCG vaccination was given to $82 \%$ of RA patients. The mean age of the Healthy Controls was $38.75 \pm 7.26$ yrs and BCG vaccination was given to $88 \%$ of the individuals. The mean age of TB patients being $34 \pm 15.64$ yrs. (Table 1 )

\begin{tabular}{|c|c|c|c|}
\hline Characteristic & $\begin{array}{c}\text { RA } \\
\text { patients } \\
(n=38)\end{array}$ & $\begin{array}{c}\text { TB } \\
\text { patients } \\
(n=50)\end{array}$ & $\begin{array}{c}\text { Healthy } \\
\text { Controls }(n=50)\end{array}$ \\
\hline Age, mean \pm SD yrs & $45 \pm 12.12$ & $34 \pm 15.64$ & $38.75 \pm 7.26$ \\
\hline BCG Vaccination, $\%$ & $82 \%$ & $69 \%$ & $88 \%$ \\
\hline History of TB, \% & $39.40 \%$ & - & - \\
\hline Disease Duration, mths & 53.6 mths & - & - \\
\hline RF Positivity, \% & $71.40 \%$ & - & - \\
\hline DAS28 CRP, mean \pm SD & $4.9 \pm 0.97$ & - & - \\
\hline Methotrexate, $\%$ & $42.10 \%$ & - & - \\
\hline Leflunomide, \% & $15.80 \%$ & - & - \\
\hline Hydroxychloroquine, \% & $65.80 \%$ & - & - \\
\hline Sulfasalazine, $\%$ & $13.20 \%$ & - & - \\
\hline TST + ve, $\%$ & $28.57 \%$ & $91 \%$ & $58.33 \%$ \\
\hline QFT-GIT +ve, \% & $39.29 \%$ & $87.50 \%$ & $50 \%$ \\
\hline Elispot +ve, \% & $42.85 \%$ & $84.30 \%$ & $52 \%$ \\
\hline
\end{tabular}

BCG: Bacillus Calmette-Guerin; RF: Rheumatoid Factor; DAS: Disease Activity Score; Active disease > 3.2;TST: Tuberculin Skin Test; QFTGIT: QuantiFeron TB Gold in tube

$71.4 \% \%$ of RA patients were rheumatoid factor positive, with a mean disease duration of 53.6 months The mean DAS28 CRP score was $4.9 \pm 0.97 ; 94 \%$ of RA patients had active disease. 42.1 $\%$ of the patients were receiving treatment with Methotrexate, $15.8 \%$ were treated with Leflunomide $65.8 \%$ were treated with Hydroxychloroquine and $13.2 \%$ were being treated with Sulphasalazine. No patient had received an anti-TNF $\alpha$ agent or other biological treatment.

\section{TST and IGRAs}

The mean TST diameter was $8.11 \mathrm{~mm}$ in RA patients and 13 $\mathrm{mm}$ in Controls. This was also reflected by lower proportion of positive TST results in RA group (28.57\%), as compared with controls (58.33\%). Around 91\% of TB patients were TST positive. For the QFT-GIT test, $39.29 \%$ of RA patients had a positive result, a lower rate than that for the controls (50\%). The QFT-GIT positivity in TB patients was $87.5 \%$ and by Elispot assay is $84.3 \%$. $42.85 \%$ of RA patients were positive by Elispot assay while $52 \%$ 
of the controls were positive by the Elispot assay. The RA patients have slightly lower Elispot positive rate than the Controls.

Overall, the rate of TST positivity in RA patients was only $49 \%$ of that for the Controls, lower than for the IGRAs. The positivity rate of QFT-GIT for RA patients was 78\% of that for the Controls. For Elispot assay, the positivity rate for RA patients was $82 \%$ of that for the Controls.

Table 2 shows that around $63.64 \%$ of the RA patients had normal x-ray findings and $36.36 \%$ had abnormal X-ray findings suggesting of latent TB infection in RA patients. About $94.44 \%$ of healthy controls showed normal x-ray findings. The x-rays of all TB patients suggest active pulmonary TB. (Table 2)

Table 2: Chest X-Ray findings in the study Participants

\begin{tabular}{|c|c|c|c|}
\hline Chest X-ray & $\begin{array}{c}\text { RA patients } \\
\text { (n=38) }\end{array}$ & $\begin{array}{c}\text { TB patients } \\
\mathbf{( n = 5 0 )}\end{array}$ & $\begin{array}{c}\text { Healthy Controls } \\
\mathbf{( n = 5 0 )}\end{array}$ \\
\hline Normal & $63.64 \%$ & Nil & $94.44 \%$ \\
\hline Suggestive LTBI & $36.36 \%$ & Active TB & $5.56 \%$ \\
\hline
\end{tabular}

As seen in Table 3, the overall agreement between TST and QFT-GIT was low moderate in RA patients and a moderate agreement in Healthy Controls (60.71\% vs. $70.83 \%$ ). TST +ve / QFT- ve results were similar in RA patients and Controls $(18.4 \%$ vs. 16\%); also the TST -ve/ QFT + ve result was higher in RA patients compared with that in Controls $(21.1 \%$ vs. $12 \%)$. The agreement between the two IGRAs i.e., between QFT-GIT and Elispot assay is moderate (78.5\%; $\mathrm{k}=0.593)$. (Table 3)

As seen in Table 4, the overall agreement between TST and Elispot assay was low moderate in RA patients and a moderate agreement in Healthy Controls (53.57\% vs. $79.17 \%$ ). TST +ve / Elispot -ve results were similar in RA patients and Controls $(10.5 \%$ vs. $8 \%)$; the TST -ve /Elispot +ve result was higher in RA patients compared with that in Controls (21.1\% vs. $12 \%)$. (Table 4)

From Table 5, it is observed that the sensitivity of QFT-GIT and Elispot assay is $55.56 \%$ and $52.9 \%$ respectively and the specificity of the QFT-GIT and Elispot assay is $92.3 \%$ and $85.7 \%$ respectively according to the latent TB screening using abnormalities in the

Table 3: Agreement between QFT-GIT and TST in Rheumatoid Arthritis patients and Healthy Controls ( $\mathrm{n}=38$ )

\begin{tabular}{|c|c|c|c|c|c|}
\hline & QFT -GIT Positive (\%) & QFT-GIT Negative (\%) & Total & Agreement (\%) & kappa \\
\hline RA: TST Positive & $7(18.4)$ & $7(18.4)$ & $14(36.8)$ & \multirow{2}{*}{60.71} & \multirow{2}{*}{0.313} \\
\hline TST Negative & $8(21.1)$ & $16(42.1)$ & $24(63.2)$ & & \\
\hline HC: TST Positive & $21(42)$ & $8(16)$ & $29(58)$ & \multirow{2}{*}{70.83} & \multirow{2}{*}{0.408} \\
\hline TST Negative & $6(12)$ & $15(30)$ & $21(42)$ & & \\
\hline
\end{tabular}

QFT-GIT: QuantiFeron TB Gold in tube; TST: Tuberculin Skin Test;

RA: Rheumatoid Arthritis; HC: Healthy Controls

Table 4: Agreement between Elispot and TST in Rheumatoid Arthritis patients and Healthy Controls $(n=38)$

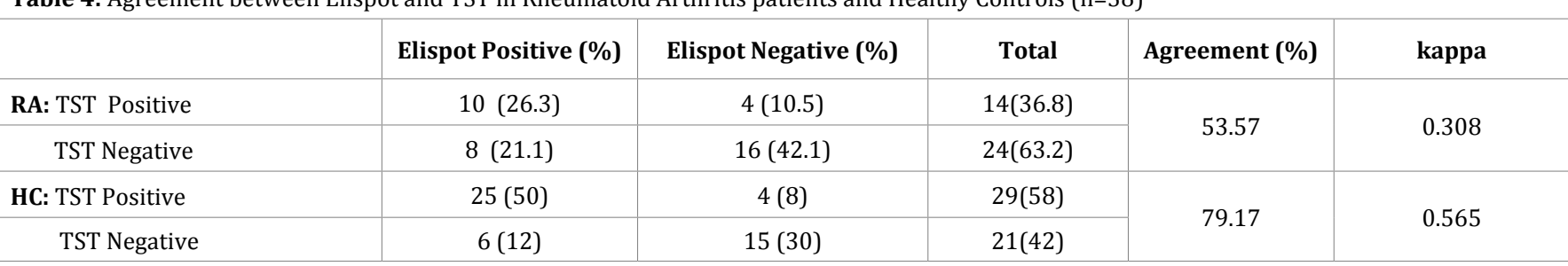

TST: Tuberculin Skin Test; RA: Rheumatoid Arthritis; HC: Healthy Controls

Table 5: Comparison of IGRAs: QFT-GIT ELISA and Elispot assay with latent TB screening in RA patients $(n=38)$

\begin{tabular}{|c|c|c|}
\hline Test & Sensitivity & Specificity \\
\hline TST & $37.50 \%$ & $75 \%$ \\
\hline QFT-GIT Elisa & $55.56 \%$ & $92.30 \%$ \\
\hline Elispot assay & $52.90 \%$ & $85.70 \%$ \\
\hline
\end{tabular}

chest x-ray and having history of tuberculosis. The sensitivity of TST and IGRA was equally low but the specificity of IGRA was higher. (Table 5)

Figure 2 shows that the positivity of Elispot assay and QFTGIT is lower than TST in Healthy Controls. The TST positivity is $58.33 \%$ compared to $52 \%$ and $50 \%$ for Elispot assay and QFT-GIT assay respectively. (Figure 2)

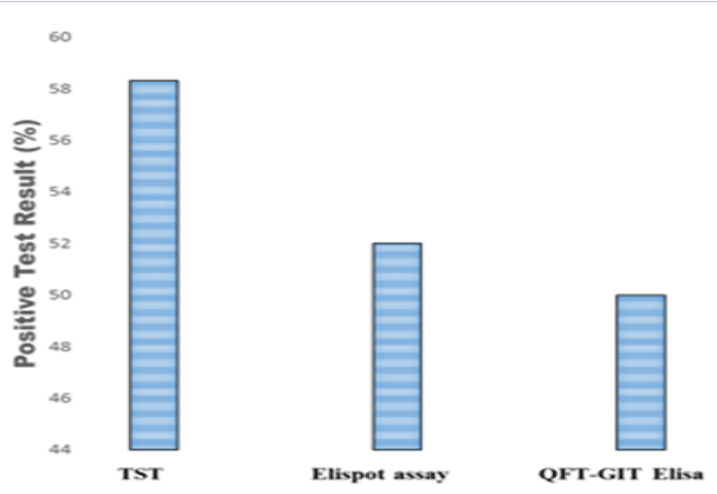

Figure 2: Comparison of TST and IGRAs positivity in Healthy Controls $(n=50)$

Citation: Sudha SD, Shradha B, Arun C, Jyostna J (2018) Utility of Interferon Gamma Assays and Comparison with Tuberculin Skin Test in Rheumatoid Arthritis Patients. J Rheumatol Arthritic Dis 3(2): 1-8. 


\section{Discussion}

Traditional TST is one of the most widely used TB screening methods with advantages of convenient application and low cost. However, it has several disadvantages, such as high falsepositivity rate and low specificity due to cross-reactions with antigen of Mycobacterium bovis BCG strain and non-tuberculosis mycobacteria, false-negativity rate and low sensitivity due to the state of immunosuppression, which limit the value of TST diagnosis in rheumatic diseases patients. [19] Our study suggests that the IGRAs maintained better performance in the study participants than the TST.

India, being a TB burden country, our patients are at high risk of TB. The number of patients who tested positive was more comparable between RA patients and controls with QFTGIT (39.29\% vs. $50 \%$ ) and Elispot assay ( $42.85 \%$ vs. $52 \%$ ), whereas the TST detected less LTBI among patients with RA than controls ( $28.57 \%$ vs. $58.33 \%)$. These findings suggest that the sensitivity of the QFT-GIT for the diagnosis of LTBI could be higher than that of the TST in patients with RA, even in cases of RA immunosuppression. To our knowledge, this is the first study in indian population to determine the agreement between TST and IGRAs in RA patients, TB patients and Healthy Controls.

The TST positivity rate in controls in this study commensurate with high prevalence of LTBI among the general population of our country. [11] Also, the TST result in RA patients is in concordance with the findings, in a TB-endemic population by Ponce de Leon et al [9] They reported that the TST is severely compromised in patients with RA. $26.7 \%$ of RA patients in their study were TST positive and $65.6 \%$ of the controls were TST positive.

It has been suggested that the IFN gamma assays may be less affected by immunosuppression than the TST in the RA population. Ewer et al has showed the interference of BCG vaccination with a positive TST.[20] The TST positivity in the control group in this study might explain the related interference. It was shown that BCG vaccination increase the likelihood of false-positive TST results for upto 15 years. A study by Cobanoglu et al showed that when comparing subjects under 25 years of age to subjects aged older than 25 years, repeated BCG vaccinations were related to high discordance between the TST and QFT-G in subjects older than 25 years. [21]

QFT-GIT test results are shown to be independent from BCG vaccination, it is not influenced by the disease activity, and it seems to be significantly more accurate than the skin test for the diagnosis of LTBI. [22]QGT-G In Tube (QFT-GIT) showed less indeterminate results as compared to QFT-Gold test. Takahashi et al reported in 14 patients only $5 \%$ of QFT tests were indeterminate. [23] TST is dependent on a normal T-cell function, and a negative TST result may either rule out LTBI or reflect abnormal delayed-type hypersensitivity (DTH) due to deficient cell-mediated immunity. IFN gamma assays are based on the production of IFN gamma by mononuclear cells exposed to TB antigens. Peripheral T-cells from RA patients stimulated with phytohaemagglutinin or anti-CD3 are reported to have produced significantly less interferon response than T-cells from healthy controls. [8]

It has been shown that TST reaction is suppressed in patients with RA.[24, 25] Conflicting evidence has been reported concerning impaired cell mediated immunity in RA patients, which may be due to the disease itself or to immunosuppressive drugs. [8] In our study, four patients of RA showed no reaction to TST. A high number of patients showed no TST reaction compared to healthy controls in a study by Inanc et al, supporting the limitations of TST, especially in immuno compromised patients. [26] Immuno compromised patients are often anergic, and results of previous studies have suggested that the rate of TST false-negative results is $\sim 40 \%$ in patients with RA.[27]

In our study, overall agreement between TST and IGRAs in healthy subjects was high as compared to the RA patients. The small sample size can be considered as a limitation in our study. There was a low moderate agreement observed between IGRAs and TST in RA patients while a moderate agreement was seen in healthy controls between the tests. In our study, the sensitivity of TST and IGRAs was equally low but the specificity of IGRAs was higher. The specificity of the QFT-GIT and Elispot assay is 92.3 $\%$ and $85.7 \%$ respectively according to the latent TB screening using abnormalities in the chest x-ray and having history of tuberculosis. Mariette et al [17] reported that the sensitivity of TST was low as compared to TST but the specificity of IGRA was higher with regard to the 57 patients considered to have LTBI on questioning or $\mathrm{x}$-ray independently from the tests. A recent metaanalysis showed that QFT-G has high specificity (96\%) whereas TST has low and highly variable specificity in BCG-vaccinated populations. $[28,29]$ Two main factors are known to affect TST specificity: environmental mycobacteria and BCG vaccination. [30] In our study, 87.5\% TB patients were positive by QFT-GIT assay and $84.3 \%$ patients were positive by Elispot assay. In a study conducted by James et al in patients with TB patients in India, the QFT-IT was positive in all patients with active TB. [7] Lee et al also reported that T-SPOT and QFT-G assay demonstrated superior specificity to TST in a cohort of patients with active TB infection. [31] In our study, a moderate agreement was seen in healthy controls between the TST and IGRAs. Kabeer et al [32] conducted a study in indian population which included 186 healthy controls and 200 healthy household contacts. In this study, the agreement between TST and QFT-IT was moderate $(\mathrm{k}=0.490)$. A previous study conducted using ESAT- 6 and CFP-10 based ELISPOT in the Mumbai city reported that $80 \%$ of healthy adults (age range 1870; mean 47 years) were positive for $M$. tuberculosis infection. [32]

The differences between IGRAs and TST results are reported in various studies. Chapman et al [33] compared invitro assay with TST in Zambian individuals. Sellam et al [34] found that the IGRA was significantly less affected by immunosuppression than the TST in patients with RA. Matulis et al [35] reported that QFTGIT results were significantly associated with the presence of LTBI risk factors in patients with auto-immune diseases receiving immunosuppressive drugs, including anti-TNF alpha inhibitors.

Studies show that positive results on the IGRA have a greater 
association with TB risk factors than positive results on the TST, suggesting that the IGRA has better sensitivity and specificity than the TST.[27] The limiting factor for the use of IGRAs in high burden, resource limited countries are the high material costs and the need for laboratory infrastructure and trained personnel.[12] Martin et al evaluated 150 patients with inflammatory arthritis with a minimum BCG vaccination rate of $82 \%$. They reported a concordance between the two IFN- $\gamma$ assays of $98.4 \%$. Kleinert et al have studied 90 rheumatic patients.[36] The agreement between the three tests were of $83.1 \%(\mathrm{k}=0.44)$. Bocchino et al. compared the performance of two commercial interferon IFN- $\gamma$ assays: TSPOT.TB and QFT-GIT with TST for the detection of LTBI in patients with RA, psoriatic arthritis and inflammatory bowel disease, and concluded that INF- $\gamma$ assays may confirm positive TST results and reveal additional cases of LTBI in selected patients, when TST false-negative results are suspected.[37]

In conclusion, TST response is suppressed in patients treated with immunosuppressive drugs, and there is low moderate agreement between the IGRAs and TST in patients with inflammatory diseases. Clinicians should pay particular attention to immunosuppressed patients with negative TST and indeterminate IGRA test results and always consider individual clinical factors more heavily than equivocal test results. Our study shows that the IGRAs are more accurate than the TST in diagnosing latent TB infection among a high risk population of RA patients. Several screening algorithms have already been proposed. One of them has suggested replacing TST with a dual IGRA strategy (Elispot assay and QFT-G). Another dual testing strategy based on both TST and IGRA results has also been proposed.[30] However further studies are needed to validate these strategies.

\section{Acknowledgements}

The authors wish to thank: The Director and the Management of Sir H. N. Medical Research Society for the Funds, the Scientific Advisory Committee of Sir H. N. Reliance Foundation Hospital and Research Centre for sanctioning the Project and the patients for participating in this project. Special thanks to Department of Chest Medicine, BYL Nair Hospital for their patient's recruitment and collaboration for supporting this project and Hinduja Hospital, Mumbai, Microbiology department for the special diagnostic test.

\section{Contributions}

Ms. Shradha Borukar: Conducting the necessary tests, analysis of the data and writing manuscript.

Dr. Arun Chogle: Principal Investigator and Conception of the project, recruitment of the patients and excellent clinical inputs during the conduct of the study.

Dr. Jyostna Joshi: Co-Investigator, recruitment of the patients and excellent clinical inputs during the conduct of the study.

Dr. Sudha S Deo: Co-Principal Investigator, Conception of the project and finalizing the manuscript.

\section{Conflict of Interest}

There is no conflict of interest between any of the authors.

\section{References}

1. Jagtap S, Turankar A V, Bankar M A, Jaiswal K M, V. M Motghare, Urade C, et al. Status of Serum Vitamin D Levels In Patients of Rheumatoid Arthritis: A Pilot Study. J Rational Pharmacother Res. 2014;2(3): 6566.

2. Tembe A G, Kharbanda P, Bhojani K, Joshi V R. Profile of rheumatoid arthritis patients attending a private tertiary hospital rheumatology clinic. Indian Journal of Rheumatology. 2008;3(4): 144-147. doi: 10.1016/S0973-3698(10)60140-9

3. Mehta B, Zapantis E, Petryna O, Efthimiou P. Screening Optimzation of Latent Tuberculosis Infection in Rheumatoid Arthritis Patients. Arthritis.2015; 2015(2015): 1-8. doi: 10.1155/2015/569620

4. Lee JH, Sohn HS, Chun JH, Hyoun-AK Chang HS, Yun WL, et al. Poor agreement between QuantiFERON-TB Gold test and tuberculin skin test results for the diagnosis of latent tuberculosis infection in rheumatoid arthritis patients and healthy controls. Korean J Intern Med. 2014;29(1):76-84. doi: 10.3904/kjim.2014.29.1.76

5. Matsumura R, Igari H, Nakazawa T, Ishikawa S, Tsuyuzaki M, Suzuki K, et al. Comparative utility of interferon $\gamma$ release assay, QuantiFERON® TB-GIT and T-SPOT®.TB in rheumatoid arthritis. Int J Tuberc Lung Dis. 2016; 20(11): 1546-1553. doi: 10.5588/ijtld.16.0038

6. Sharma SK, Vashishtha R, Chauhan LS, Sreenivas V, Seth D. Comparison of TST and IGRA in Diagnosis of Latent Tuberculosis Infection in a High TB-Burden Setting. Plos One. 2017; 12(1):e0169539. doi: 10.1371/ journal.pone.0169539

7. James PM, Ganaie FA, Kadahalli RL. The performance of QuantiferonTB Gold in-Tube (QFT-IT) test compared to Tuberculin Skin Test (TST) in detecting latent tuberculosis infection (LTBI) in the presence of HIV coinfection in a high TB-burden area with BCG-vaccinated population. J Int Assoc Provid AIDS Care. 2014; 13(1):47-55. doi: $10.1177 / 2325957412469687$

8. Shovman O, Anouk M, Vinnitsky N, Arad U, Paran D, Litinsky I, et al. QuantiFERON-TB Gold in the identification of latent tuberculosis infection in rheumatoid arthritis: a pilot study. Int J Tuberc Lung Dis. 2009; 13(11): 1427-1432.

9. Leon DP, Vasquez EA, Alvizuri S, Gutierrez C, Cucho M, Alfaro J, et al. Comparison of an interferon-r assay with tuberculin skin testing for detection of tuberculosis (TB) infection in patients with rheumatoid arthritis in a TB-endemic population. J Rheumatol. 2008; 35(5):776781.

10. Lee H, Park HY, Jeon K, Jeong BH, Hwang JW, Lee J, et al. QuantiFERONTB Gold In-Tube Assay for Screening Arthritis Patients for Latent Tuberculosis Infection before Starting Anti-Tumor Necrosis Factor Treatment. Plos One. 2015; 10(3): e0119260. doi: 10.1371/journal. pone. 0119260

11. Pai M, Denkinger CM, Kik SV, Rangaka MX, Zwerling A, Oxlade O, et al. Gamma Interferon Release Assays for Detection of Mycobacterium tuberculosis Infection. Clin Microbiol Rev. 2014; 27(1): 3-20. doi: 10.1128/CMR.00034-13

12. Martin J, Walsh C, Gibbs A, McDonnell T, Fearon U, Keane J, et al. Comparison of interferon \{gamma\} release assays and conventional screening tests before tumour necrosis factor \{alpha\} blockade in patients with inflammatory arthritis. Ann Rheum Dis. 2010; 69(1):181-185. doi: 10.1136/ard.2008.101857

13. Ruan Q Zhang S, Ai J, Shao L, Zhang W. Screening of latent tuberculosis infection by interferon- $\gamma$ release assays in rheumatic patients: a systemic review and meta-analysis. Clin Rheumatol. 2016; 35(2):417- 


\section{5. doi: 10.1007/s10067-014-2817-6}

14. Minguez S, Latorre I, Mateo L, Lacoma A, Diaz J, Olive A, et al. Interferongamma release assays in the detection of latent tuberculosis infection in patients with inflammatory arthritis scheduled for anti-tumour necrosis factor treatment. Clin Rheumatol. 2012; 31(5): 785-794. doi: 10.1007/s10067-012-1938-z

15. Hatemi G, Melikoglu M, Ozbakir F, Tascilar K, Yazici H. Quantiferon-TB Gold in tube assay for the screening of tuberculosis before and during treatment with tumor necrosis factor alpha antagonists. Arthritis Res Ther. 2012; 14(3): R147. doi: 10.1186/ar3882

16. Song G G, Bae S C, Lee Y H. Interferon-gamma release assays versus tuberculin skin testing in patients with rheumatoid arthritis. International Journal of Rheumatic Diseases. 2013; 16(3):279-283; doi: 10.1111/1756-185X.12098

17. Mariette X, Baron G, Tubach F, Lioté F, Combe B, Miceli-Richard $C$, et al. Influence of replacing tuberculin skin test with ex vivo interferon $\gamma$ release assays on decision to administer prophylactic antituberculosis antibiotics before anti-TNF therapy. Ann Rheum Dis. 2012; 71(11):1783-1790. doi: 10.1136/annrheumdis-2011-200408

18. American Thoracic Society, Centres for Disease Control and Prevention Targeted tuberculin testing and treatment of latent tuberculosis infection. Am J Respir Crit Care Med. 2000; 161(4 pt 2):S221-47

19. Jiang B, Ding H, Zhou L, Chen X, Chen S, Bao C. Evaluation of interferon-gamma release assay (T-SPOT.TB $\left({ }^{\mathrm{TM}}\right)$ ) for Diagnosis Of Tuberculosis Infection In Rheumatic Disease Patients. Int J Rheum Dis. 2016;19(1):38-42. doi: 10.1111/1756-185X.12772

20.Ewer K, Deeks J, Alvarez L, Bryant G, Waller S, Andersen P, et al Comparison of T-cell-based assay with tuberculin skin test for diagnosis of Mycobacterium tuberculosis infection in a school tuberculosis outbreak. Lancet. 2003; 361(9364):1168-1173. doi:10.1016/S01406736(03)12950-9

21. Cobanoglu N, Ozcelik U, Kalyoncu U, Ozen S, Kiraz S, Gurcan N, et al. Interferon gamma assays for the diagnosis of tuberculosis infection before using tumour necrosis factor-alpha blockers. Int J Tuberc Lung Dis. 2007; 11(11):1177-1182

22.Camlar SA, Makay B, Appak O, Appak YC, Esen N, Günay T et al. Performance of tuberculin skin test and interferon gamma assay for the diagnosis of latent tuberculosis infection in juvenile idiopathic arthritis. Clin Rheumatol. 2011; 30(9):1189-1193. doi: 10.1007/ s10067-011-1724-3

23. Takahashi H, Shigehara K, Yamamoto M, Suzuki C, Naishiro Y, Tamura Y, et al. Interferon gamma assay for detecting latent tuberculosis infection in rheumatoid arthritis patients during infliximab administration. Rheumatol Int. 2007;27(12):1143-1148. doi:10.1007/s00296-0070361-2

24.Gogus F, Günendi Z, Karakus R, Erdogan Z, Hizel K, Atalay F Comparison of tuberculin skin test and QuantiFERON-TB gold in tube test in patients with chronic inflammatory diseases living in a tuberculosis endemic population. Clin Exp Med. 2010; 10(3):173-177. doi: $10.1007 / \mathrm{s} 10238-009-0082-9$

25. Markusse HM, Hazes JM, Dijkmans BA, de Vries RR, Stanford JL. Skin test responsiveness to Mycobacterium tuberculosis, HLA-DR4, and rheumatoid arthritis. Br J Rheumatol 1988; 27:409-411
26. Inanc N, Aydin S Z, Karakurt S, Atagunduz P, Yavuz S, Direskeneli H. Agreement between Quantiferon-TB gold test and tuberculin skin test in the identification of latent tuberculosis infection in patients with rheumatoid arthritis and ankylosing spondylitis. J Rheumatol. 2009; 36(12): 2675-2681. doi: 10.3899/jrheum.090268

27. Hsia EC, Schluger N, Cush JJ, Chaisson RE, Matteson EL, Xu S, et al. Interferon- $\gamma$ release assay versus tuberculin skin test prior to treatment with golimumab, a human anti-tumor necrosis factor antibody, in patients with rheumatoid arthritis, psoriatic arthritis, or ankylosing spondylitis. Arthritis Rheum. 2012; 64(7):2068-2077. doi: $10.1002 /$ art.34382

28.Chen DY, Shen GH, Chen YM, Chen HH, Lin CC, Hsieh CW, et al. Interferon-inducible protein-10 as a marker to detect latent and active tuberculosis in rheumatoid arthritis. Int J Tuberc Lung Dis. 2011; 15(2):192-200

29.Pai M, Zwerling A, Menzies D. Systematic review: T-cell-based assays for the diagnosis of latent tuberculosis infection: an update. Ann Intern Med. 2008; 149(3): 177-184

30. Costantino F, Bittencourt M C, Rat A C, Damien L, Hervé D, Marie C B, et al. Screening for latent tuberculosis infection in patients with chronic inflammatory arthritis: Discrepancies between tuberculin skin test and interferon- $\gamma$ release assay results. The Journal of Rheumatology. 2013; 40(12): 1986-1993. doi: 10.3899/jrheum.130303

31.Lee JY, Choi HJ, Park IN, Hong SB, Oh YM, Lim CM, et al. Comparison of two commercial interferon-gamma assays for diagnosing Mycobacterium tuberculosis infection. Eur Respir J. 2006; 28(1):2430. doi: 10.1183/09031936.06.00016906

32.Syed Ahamed Kabeer B, Paramasivam P, Raja A. Interferon gamma and interferon gamma inducible protein-10 in detecting tuberculosis infection. J Infect. 2012; 64(6):573-579. doi: 10.1016/j. jinf.2012.02.013

33. Chapman A, Munkanta M, Wilkinson KA, Pathan AA, Ewer K, Ayles H, et al. Rapid detection of active and latent tuberculosis infection in HIVpositive individuals by enumeration of Mycobacterium tuberculosisspecific T cells. AIDS. 2002; 16(17):2285-2293.

34. Sellam J, Hamdi H, Roy C, Gabriel B, Marc L, Xavier P, et al. Comparison of in vitro-specific blood tests with tuberculin skin test for diagnosis of latent tuberculosis before anti-TNF therapy. Ann Rheum Dis. 2007; 66(12): 1610-1615. doi: 10.1136/ard.2007.069799

35. Matulis G, Juni P, Villiger P M, Gadola S D. Detection of latent tuberculosis in immunosuppressed patients with autoimmune diseases: performance of a Mycobacterium tuberculosis antigenspecific interferon gamma assay. Ann Rheum Dis. 2008; 67(1): 84-90. doi:10.1136/ard.2007.070789

36. Kleinert S, Kurzai O, Elias J, Marten K, Engelke C, Feuchtenberger $\mathrm{M}$, et al. Comparison of two interferon-gamma release assays and tuberculin skin test for detecting latent tuberculosis in patients with immune-mediated inflammatory diseases. Ann Rheum Dis. 2010; 69 (4):782-784. doi: 10.1136/ard.2009.113829

37. Bocchino M, Matarese A, Bellofiore B, Giacomelli P, Santoro G, Balato N, et al. Performance of two commercial blood IFN-gamma release assays for the detection of Mycobacterium tuberculosis infection in patient candidates for anti-TNF-alpha treatment. Eur J Clin Microbiol Infect Dis. 2008; 27(10): 907-913. doi: 10.1007/s10096-008-0519-1 